\title{
Reconstruction of room impulse responses over extended domains for navigable sound field reproduction
}

Fernandez Grande, Efren; Nozal, Diego Caviedes; Hahmann, Manuel; Karakonstantis, Xenofon; Verburg Riezu, Samuel Arturo

\section{Published in:}

Proceedings of International Conference on Immersive and 3D Audio

Link to article, DOI:

10.1109//3DA48870.2021.9610846

Publication date:

2021

Document Version

Peer reviewed version

Link back to DTU Orbit

Citation (APA):

Fernandez Grande, E., Nozal, D. C., Hahmann, M., Karakonstantis, X., \& Verburg Riezu, S. A. (2021).

Reconstruction of room impulse responses over extended domains for navigable sound field reproduction. In Proceedings of International Conference on Immersive and 3D Audio IEEE.

https://doi.org/10.1109//3DA48870.2021.9610846

\section{General rights}

Copyright and moral rights for the publications made accessible in the public portal are retained by the authors and/or other copyright owners and it is a condition of accessing publications that users recognise and abide by the legal requirements associated with these rights.

- Users may download and print one copy of any publication from the public portal for the purpose of private study or research.

- You may not further distribute the material or use it for any profit-making activity or commercial gain

- You may freely distribute the URL identifying the publication in the public portal 


\title{
Reconstruction of room impulse responses over extended domains for navigable sound field reproduction
}

\author{
Efren Fernandez-Grande ${ }^{a)}$ \\ Acoustic Technology, Dept. Electrical Engineering \\ Technical University of Denmark \\ Kgs. Lyngby, Denmark \\ a) efg@elektro.dtu.dk \\ Manuel Hahmann \\ Acoustic Technology, Dept. Electrical Engineering \\ Technical University of Denmark \\ Kgs. Lyngby, Denmark
}

\author{
Diego Caviedes-Nozal $\left.{ }^{b}\right)$ \\ Acoustic Technology, Dept. Electrical Engineering \\ Technical University of Denmark \\ Kgs. Lyngby, Denmark \\ ${ }^{b)}$ dicano@elektro.dtu.dk \\ Xenofon Karakonstantis \\ Acoustic Technology, Dept. Electrical Engineering \\ Technical University of Denmark \\ Kgs. Lyngby, Denmark
}

\author{
Samuel A. Verburg \\ Acoustic Technology, Dept. Electrical Engineering \\ Technical University of Denmark \\ Kgs. Lyngby, Denmark
}

\begin{abstract}
The acquisition of the spatio-temporal characteristics of a sound field over a large volume of space is experimentally challenging, as a large number of transducers is required to sample the sound field. Sound field reconstruction methods are a resourceful approach, as they enable the interpolation and extrapolation of the sound field from a limited number of observed data. In this study we examine the spatio-temporal and spatio-spectral reconstruction of the sound field in a room from distributed measurements of the sound pressure. Specifically, a variational Gaussian process regression model is formulated, using time-domain anisotropic kernels to reconstruct the direct sound and early reflections, and frequency-domain isotropic kernels for reconstructing the late reverberant field. The proposed methodology is compared experimentally to classical regression models based on plane wave decompositions, which are widely used in sound field reconstruction in enclosures due to their simplicity and accuracy.
\end{abstract}

Index Terms-Sound field reconstruction, Gaussian processes, spatial audio, 6dof, room impulse response, acoustic holography, microphone array

\section{INTRODUCTION}

The spatio-temporal acquisition of a sound field over a large volume of space is experimentally challenging, as a large number of transducers is required to sample the threedimensional field. Some approaches have been presented to effectively acquire the pressure field over a large aperture, e.g. by means of automated measurements [1], [2], IoT-enabled devices [3], by deploying large sensor networks throughout space [4], [5] or via remote sensing principles [6]-[9]. In most cases, the direct acquisition of the sound field with sufficient spatial resolution is not viable, due to excessive spatial sampling requirements. Sound field reconstruction methods are a resourceful approach, as the enable the reconstruction - via interpolation and extrapolation, of a given sound field from a set of limited observations distributed throughout space. These methods are widely used in applications such as acoustic holography [10]-[12], sound field analysis [13], [14], or active control of sound [4], [15], [16].

In recent years, advances in immersive and interactive sound field reproduction, have generated a need for navigable reproduction, allowing a listener to move throughout the sound field. To this end, various sound field reconstruction models have been proposed, both using distributed sensors or multiple arrays [17]-[22]. In the present study we add to the existing knowledge by formulating and examining a method to extrapolate the sound field in a room based on a spatiotemporal model of the Green's function in an enclosure.

The spatio-temporal reconstruction of the sound field in a room is a central problem in acoustics. It is a challenging problem due to the complex influence of boundaries, scattering and diffracting elements and the resulting spatio-temporal complexity of reverberant fields. In order to improve the reconstruction, it is common practice to incorporate available physical knowledge. Existing approaches incorporate knowledge about the modal structure of rooms at low frequencies [23]-[26], spatial sparsity of sound waves in the enclosure [2], sparsity of time impulses [27], [28], or the statistical properties of the sound field at high frequencies [29].

When considering the properties of the sound field in a room, its temporal structure is one of the most defining elements, and it is a physical process which generalizes across different rooms [30]. Namely, it consists of the distinct arrival 
of the direct field, early reflections and possibly scattered and diffracted components - in most applications, the correct reconstruction of the direct field and the early reflections is of utmost importance, as they are of particular physical and perceptual relevance. After the direct sound, the reflection density grows quadratically with time, and at a fairly early stage of the room impulse response the structure of the sound field becomes seemingly chaotic - it being stochastic in nature. The detailed modeling of this fine structure is arguably less significant than the early part, although it is still important to correctly capture the decay rate, coloration and directional energy distribution of the reverberation. Inasmuch as the process described above is a general one, it is not straightforward to include such structure in the reconstruction.

In this study we propose the reconstruction of the sound field in an enclosure based on the explicit modeling of the direct field in time domain, followed by a wave expansion of early reflections and late reverberation. We do this by posing an inverse problem, which avoids enforcing any particular structure on the reflection properties (i.e. not necessarily specular, nor homogeneous boundaries, etc.) . The physical structure adopted for the reconstruction is that of the Green's function in an enclosure, analogous to a Neumann or Dirichlet Green's function [10], [31], instead of the more commonly encountered Green's function expansion into normal modes [32]. The rationale behind this choice is that the spatiotemporal structure of the room reverberation can be incorporated more explicitly via a Neumann type Green's function, leading to a better reconstruction. The model is formulated as a Gaussian process regression [33], which enables to incorporate the spatial statistics of the sound fields, and account for the expected anisotropy of the early part, in comparison to more isotropic late response [34]. Gaussian processes enable to do this conveniently, by including structure via the second order spatial statistics of the field. The framework is also convenient for defining a hierarchical Bayesian model, which is useful for the problem at hand [33], [35], [36].

\section{THEORY}

\section{A. Sound field reconstruction model}

The sound pressure in a lightly damped room due to a point source is often expressed via the Green's function expanded into the normal modes $\Psi_{n}$ of the enclosure [32]

$$
p(\mathbf{r})=-\frac{\mathrm{j} \omega \rho Q}{V} \sum_{n=0}^{\infty} \frac{\Psi_{n}\left(\mathbf{r}_{0}\right) \Psi_{n}(\mathbf{r})}{k^{2}-k_{n}^{2}-j \omega / \tau_{n}} \mathrm{e}^{\mathrm{j} \omega t},
$$

where $\Psi_{n}$ are the normal modes of the room, $k_{n}$ are their modal wavenumbers, $\tau_{n}$ their time constants, $k$ is the wavenumber in air, $\omega$ the angular frequency, $\rho$ density of the medium, $V$ the volume of the room, $\mathbf{r}_{0}$ is the position of the source, $Q$ its volume velocity and $\mathbf{r}$ is the observation position. The sound pressure in the room and the Green's function, i.e., the solution to inhomogeneous Helmholtz equation, are related via the source volume acceleration as $p(\mathbf{r})=j \omega \rho Q G\left(\mathbf{r} \mid \mathbf{r}_{\mathbf{0}}\right)$.
An alternative and analogous expression of the Green's function in a rigid enclosure, is found via the Neumann Green's function [10], [31], [37]

$$
p(\mathbf{r})=\mathrm{j} \omega \rho Q\left(G+g_{N}\right)
$$

where $G$ is the free-field Green's function that accounts for the direct field radiated by the source

$$
G\left(\mathbf{r} \mid \mathbf{r}_{0}\right)=\frac{\mathrm{e}^{\mathrm{j} k\left\|\mathbf{r}-\mathbf{r}_{0}\right\|}}{4 \pi\left\|\mathbf{r}-\mathbf{r}_{0}\right\|}
$$

and $g_{N}\left(\mathbf{r} \mid \mathbf{r}_{0}\right)$ accounts for the influence of the boundaries i.e. in the case of Neumann boundary conditions, $g_{N}$ ensures that the normal component of the particle velocity is null, $u_{n}=0$ at the boundaries $S$ of the enclosure. If the boundary conditions were Dirichlet or Robin, a similar function can be constructed to account for the boundary conditions, leading to a Dirichlet or Robin Green's Function.

The Neumann Green's function formulation is commonly found in scattering problems, or in acoustic propagation in waveguides or above an infinite plane, but it is less commonly found in enclosed fields. There is a notable exception, the Image source method (ISM), also known as method of images, which is a particular case of the Neumann Green's function for specularly reflecting planar boundaries, and which is widely adopted in acoustics [38], [39]. In frequency domain it is

$$
p\left(\omega, \mathbf{r} \mid \mathbf{r}_{\mathbf{0}}\right)=\sum_{q=1}^{8} \sum_{r=-\infty}^{\infty} A(\omega) \frac{\mathrm{e}^{j\left(\omega t-k\left\|\mathbf{r}_{\mathbf{q}}+\mathbf{r}_{\mathbf{r}}\right\|\right)}}{4 \pi\left\|\mathbf{r}_{\mathbf{q}}+\mathbf{r}_{\mathbf{r}}\right\|}
$$

and in the time domain,

$$
p\left(t, \mathbf{r} \mid \mathbf{r}_{\mathbf{0}}\right)=\sum_{q=1}^{8} \sum_{r=-\infty}^{\infty} A(t) * \frac{\delta\left(t-\left\|\mathbf{r}_{\mathbf{q}}+\mathbf{r}_{\mathbf{r}}\right\| / c\right)}{4 \pi\left\|\mathbf{r}_{\mathbf{q}}+\mathbf{r}_{\mathbf{r}}\right\|}
$$

where $\mathbf{r}_{\mathbf{q}}$ are the 8 vectors corresponding to the permutations of $\left(x_{0} \pm x, y_{0} \pm y, z_{0} \pm z\right), r$ is the integer vector triplet $(m, n, l)$ and $\mathbf{r}_{\mathbf{r}}=2\left(m L_{x}, n L_{y}, l L_{z}\right)$ [39]. It should be noted that this solution is a geometrical one, as reflections are required to be specular, and the formulae are not strictly valid in most ordinary rooms. The ISM is very powerful for its simplicity and physical tractability, yet it can be a restrictive model for inverse problems, because it imposes a strong physical structure on the sound field. Contrarily, the more general form of Eq. (2) does not assume specular reflections nor planar boundaries, and thus is less restrictive.

In the present study, we do not assume a closed-form of $g_{N}$. Instead an inverse problem is formulated, where the function $g_{N}$ is modeled by elementary wave expansions (without 
explicit assumptions on the nature of the reflections). The formulated model is

$$
\begin{array}{r}
p(t, \mathbf{r})=\sum_{d=1}^{D} Q_{d}(t) * \frac{\delta\left(t-\left\|\mathbf{r}-\mathbf{r}_{d}\right\| / c\right)}{4 \pi\left\|\mathbf{r}-\mathbf{r}_{d}\right\|} \\
+\left.\sum_{s=1}^{S} A_{s}(t) * \frac{\delta\left(t-\left\|\mathbf{r}-\mathbf{r}_{s}\right\| / c\right)}{4 \pi\left\|\mathbf{r}-\mathbf{r}_{s}\right\|}\right|_{\left\|\mathbf{r}_{s}\right\|=r_{\text {early }}} \\
+\left.\sum_{j=1}^{J} B_{j}(t) * \delta\left(t-\left\langle\mathbf{r}, \mathbf{r}_{j}\right\rangle / c\right)\right|_{\left\|\mathbf{r}_{j}\right\|=r_{\text {late }}}
\end{array}
$$

The first term corresponds to the direct field, which is expressed as an equivalent source model, with equivalent sources of volume velocities $Q_{d}$ [ [40], [41]]. This enables to model source directivities that may not be monopolar (it is a similar approach as presented in Ref. [42] where the source radiation properties are expanded into spherical harmonics. The effect of boundaries is introduced via the second and third terms in Eq. (6) where $A_{s}$ are the time-dependent amplitudes of a set of point sources located on a sphere of radius $r_{\text {early }}$, which account for the early part of the room impulse response in time domain. Finally, the last term of the model corresponds to a plane wave expansion, which serves to model the late part of the decay. It consists of plane waves with amplitudes $B_{j}$, each of them scaled at plane $\mathbf{r}_{j}$ and their propagation through space given by the inner product $\left\langle\mathbf{r}, \mathbf{r}_{j}\right\rangle$. It is of particular convenience to expand the later reverberation based on the plane-wave terms $B_{j}$, using the second order spatial statistics of isotropic random wave fields, as discussed in the following sub-section (Sect. II-B).

It should be noted that Eq. (6) follows a structure analogous to the Neumann (or also Dirichlet) Green's Functions, $G_{N}=$ $G+g_{N}$, where $G$ is the free-field Green's function and $g_{N}$ is a non-zero homogeneous solution of the Helmoltz equation, which accounts for the boundary conditions of the problem [10]. In the present study these boundary conditions need not be Neumann, as no assumptions are made on the reflection at the boundaries.

\section{B. Gaussian process methodology}

Consider the problem of reconstructing a sound field in a room from a limited set of measurements $\tilde{\mathbf{p}}=$ $\left[\tilde{p}\left(t_{1}, \mathbf{r}_{1}\right), \ldots, \tilde{p}\left(t_{N}, \mathbf{r}_{1}\right), \ldots, \tilde{p}\left(t_{N}, \mathbf{r}_{M}\right)\right]$. The measured pressure $\tilde{p}(t, \mathbf{r})$ at a location $\mathbf{r}$ and time $t$ is

$$
\tilde{p}(t, \mathbf{r})=p(t, \mathbf{r})+e(t, \mathbf{r})
$$

where $e(t, \mathbf{r})$ is measurement noise and $p(t, \mathbf{r})$ is the unknown function that defines the sound field, and we use Gaussian process regression to find it [33].

The sound field in the room is considered a zero mean Gaussian process

$$
p(t, \mathbf{r}) \sim \mathcal{G P}\left(0, \kappa\left(t, \mathbf{r}, t^{\prime}, \mathbf{r}^{\prime}\right)\right)
$$

where $\mathcal{G} \mathcal{P}$ stands for Gaussian process, and $\kappa\left(t, \mathbf{r}, t^{\prime}, \mathbf{r}^{\prime}\right)$ is the kernel (which is a spatio-temporal correlation function), between the sound field at $(t, \mathbf{r})$ and $\left(t^{\prime}, \mathbf{r}^{\prime}\right)$

$$
\kappa\left(t, \mathbf{r}, t^{\prime}, \mathbf{r}^{\prime}\right)=\mathbb{E}\left[p(t, \mathbf{r}) p\left(t^{\prime}, \mathbf{r}^{\prime}\right)\right]
$$

If the measurement noise is also Gaussian, such that

$$
e(t, \mathbf{r}) \sim \mathcal{G} \mathcal{P}\left(0, \kappa_{e}\left(t, \mathbf{r}, t^{\prime}, \mathbf{r}^{\prime}\right)\right)
$$

the predictive sound field function $p_{\bullet}(t, \mathbf{r})$ is the outcome of a Gaussian process given the measured pressure $\tilde{\mathbf{p}}$ [33]

$$
p_{\bullet}(t, \mathbf{r}) \mid \tilde{\mathbf{p}} \sim \mathcal{G P}\left(\mu_{p_{\bullet} \mid \tilde{\mathbf{p}}}(\mathbf{r}, t), \kappa_{p_{\bullet} \mid \tilde{\mathbf{p}}}\left(t, \mathbf{r}, t^{\prime}, \mathbf{r}^{\prime}\right)\right)
$$

where the subscript $(\cdot)$ • denotes predictions. The optimal sound field reconstruction is the posterior mean

$$
\mu_{p_{\bullet} \mid \tilde{\mathbf{p}}}(t, \mathbf{r})=\boldsymbol{\kappa}^{\mathrm{T}}(\mathbf{K}+\boldsymbol{\Sigma})^{-1} \tilde{\mathbf{p}}
$$

where $\kappa$ are the kernels (spatio-temporal correlations) between the $M$ measured pressures $\boldsymbol{\kappa}=\left[\kappa\left(t_{1}, \mathbf{r}_{1}, t, \mathbf{r}\right) \cdots \kappa\left(t_{N}, \mathbf{r}_{M}, t, \mathbf{r}\right)\right]$, and the covariance matrices $\mathbf{K}$ and $\boldsymbol{\Sigma}$ are

$$
\begin{aligned}
& \boldsymbol{\Sigma}=\mathbb{E}\left[\mathbf{e e}^{\mathrm{T}}\right], \\
& \mathbf{K}=\mathbb{E}\left[\mathbf{p p}^{\mathrm{T}}\right] .
\end{aligned}
$$

In order to interpolate and extrapolate the sound field using Eq. (12), the kernel function $\kappa$ has to be defined.

\section{Spatio-temporal kernels}

In Gaussian process regression, a kernel is a positive semidefinite function which is used to construct the covariance of the studied field, i.e. the spatio-temporal correlation of the sound field in the present case. In sound field reconstruction, it is common to assume that the sound field function $p(t, \mathbf{r})$ can be well approximated by a linear combination of elementary functions

$$
p(t, \mathbf{r}) \simeq \sum_{l=1}^{L} \phi_{l}(t, \mathbf{r}) * w_{l}(t)
$$

where $\phi_{l}(t, \mathbf{r})$ is the elementary function, frequently plane waves, spherical waves, spherical harmonics, etc., and $w_{l}(t)$ are unknown temporal weights that shape the amplitude and delay of the function. If discretized, the convolution in Eq. (14) can be made explicit as a double summation

$$
p\left(t_{n}, \mathbf{r}_{m}\right) \simeq \sum_{l=1}^{L} \sum_{i=1}^{N} \phi_{l}\left(t_{n}-t_{i}, \mathbf{r}_{m}\right) w_{l}\left(t_{i}\right)
$$

We assume that the weights $w_{l}(t)$ are also a Gaussian process such that

$$
w_{l}\left(t_{i}\right) \sim \mathcal{G} \mathcal{P}\left(0, \sigma_{l, i}^{2}\right)
$$


which implies that the elementary functions are uncorrelated (i.e. $\mathbb{E}\left[w_{l}\left(t_{i}\right) w_{l^{\prime}}\left(t_{i^{\prime}}\right)\right]=0$, if $i \neq i^{\prime}$ and $\left.l \neq l^{\prime}\right)$. From Eq. (15), the kernel in Eq. (9) in discrete form is

$$
\begin{aligned}
& \kappa\left(t_{n}, \mathbf{r}_{m}, t_{n^{\prime}}, \mathbf{r}_{m^{\prime}}\right)=\mathbb{E}\left[p\left(t_{n}, \mathbf{r}_{m}\right) p\left(t_{n^{\prime}}, \mathbf{r}_{m^{\prime}}\right)\right] \\
& =\sum_{l=1}^{L} \sum_{i=1}^{N} \sum_{l^{\prime}=1}^{L} \sum_{i^{\prime}=1}^{N} \mathbb{E}\left[w_{l}\left(t_{i}\right) w_{l^{\prime}}\left(t_{i^{\prime}}\right)\right] \\
& \quad \times \phi_{l}\left(t_{n}-t_{i}, \mathbf{r}_{m}\right) \phi_{l^{\prime}}\left(t_{n^{\prime}}-t_{i^{\prime}}, \mathbf{r}_{m^{\prime}}\right) \\
& =\sum_{l=1}^{L} \sum_{i=1}^{N} \sigma_{l, i}^{2} \phi_{l}\left(t_{n}-t_{i}, \mathbf{r}_{m}\right) \phi_{l}\left(t_{n^{\prime}}-t_{i}, \mathbf{r}_{m^{\prime}}\right) .
\end{aligned}
$$

Thus, the kernel $\kappa$ is fully defined when the variances $\sigma_{l, i}^{2}$ in Eq. (16) are determined. The elementary functions $\phi_{l}(t)$ used in this work are defined in Eq. (6), and the coefficients $w_{l}\left(t_{i}\right)$ are a generalization of the specific responses $Q_{d}(t), A_{s}(t)$ and $B_{j}(t)$.

The total amount of waves in the model is $L=D+S+J$ : The direct field is modeled by $D$ spherical sources clustered around the position of the source. The early reflections (as well as scattering and other early components) are modeled by $S$ spherical sources distributed outside the reconstruction domain similar to ESM [41]. These kernels become highly anisotropic, as they model very directional components of the sound field, and the energy flows in a few definite directions. The later part of the response is modeled by $J$ plane waves. As the reflection density grows, the later part of the response becomes more isotropic, as energy starts to flow in many more directions, and the spatial correlation of the field tends towards the well-known [32],

$$
\kappa\left(\mathbf{r}, \mathbf{r}^{\prime}\right)=\sigma^{2} \frac{\sin (k r)}{k r}
$$

which is the spatial correlation between any two points $\mathbf{r}$ and $\mathbf{r}^{\prime}$, (with $r=\left\|\mathbf{r}-\mathbf{r}^{\prime}\right\|$ ), in a random wave field, and $\sigma^{2}$ is a variance factor scaling the amplitude of the kernel [33]. This kernel is the zeroth order spherical Bessel function $j_{0}(k r)$ in three-dimensions, and is often referred to as the Bessel kernel or diffuse-field kernel.

\section{Kernel parameter estimation}

The parameters $\boldsymbol{\sigma}=\left[\sigma_{1,1}, \ldots, \sigma_{N, L}\right]$ are estimated via Bayes theorem

$$
\pi(\mathbf{p}, \boldsymbol{\sigma} \mid \tilde{\mathbf{p}})=\frac{\pi(\tilde{\mathbf{p}} \mid \mathbf{p}, \boldsymbol{\sigma}) \pi(\mathbf{p} \mid \boldsymbol{\sigma}) \pi(\boldsymbol{\sigma})}{\pi(\tilde{\mathbf{p}})},
$$

The likelihood $\pi(\mathbf{p}, \boldsymbol{\sigma})$, the conditional prior $\pi(\mathbf{p} \mid \boldsymbol{\sigma})$ and the evidence $\pi(\tilde{\mathbf{p}}$ are multivariate normal distributions calculated from Eqs. (8)-(10). The prior distribution $\pi(\boldsymbol{\sigma})$ is defined an inverse gamma, according to sparse Bayesian learning principles [33]

$$
\sigma_{i, l} \sim \operatorname{InvGamma}(a, b) .
$$

Long tailed distributions as the inverse gamma place most of the probability density close to zero, which is equivalent to assuming that a priori the variances $\sigma_{i, l}^{2}$ are very small and consequently, the coefficients $w_{i, l}$ in Eq. (16) are mostly likely to be zero (i.e. sparsity is promoted). However, the long tail of the inverse gamma still allows for the activation of those sources that have a greater impact in the reconstruction.

The posterior distribution usually does not present a closed form solution. In this study we use stochastic variational inference (SVI) to approximate the posterior. In SVI, one defines a family of candidate parameterized distributions such that $\pi_{\theta}(\mathbf{p}, \boldsymbol{\sigma} \mid \tilde{\mathbf{p}}) \approx \nu_{\phi}(\mathbf{p}, \boldsymbol{\sigma})$, where $\theta$ are hyperparameters of the posterior and $\phi$ the hyperparameters of the variational approximation. SVI approximates the posterior of the Bayes theorem via maximization of the expected lower bound (ELBO), equivalent to minimizing the Kullback-Leibler (KL) divergence [43]

$$
\begin{aligned}
\mathrm{KL}\left(\nu_{\phi}(\mathbf{p}, \boldsymbol{\sigma}) \| \pi_{\theta}(\mathbf{p}, \boldsymbol{\sigma} \mid \tilde{\mathbf{p}})\right) & =\mathbb{E}_{\nu_{\phi}}\left[\log \frac{\nu_{\phi}(\mathbf{p}, \boldsymbol{\sigma})}{\pi_{\theta}(\mathbf{p}, \boldsymbol{\sigma} \mid \tilde{\mathbf{p}})}\right] \\
& =\log \pi_{\theta}(\tilde{\mathbf{p}})-\mathrm{ELBO},
\end{aligned}
$$

which is a measure of the difference between two distributions. The ELBO is

$$
\mathrm{ELBO}=\mathbb{E}_{\nu_{\phi}}\left[\log \pi_{\theta}(\mathbf{p}, \boldsymbol{\sigma} \mid \tilde{\mathbf{p}})-\log \nu_{\phi}(\mathbf{p}, \boldsymbol{\sigma})\right]
$$

and is tractable. The proposed variational distributions $\nu_{\phi}(\mathbf{p}, \boldsymbol{\sigma})=\nu_{\phi}(\mathbf{p} \mid \boldsymbol{\sigma}) \nu_{\phi}(\boldsymbol{\sigma})$ are normal and inverse gamma respectively

$$
\begin{aligned}
& \nu_{\phi}(\mathbf{p} \mid \boldsymbol{\sigma})=\mathcal{N}\left(\boldsymbol{\mu}_{\nu}, \boldsymbol{\sigma}_{\nu}\right) \\
& \nu_{\phi}\left(\sigma_{i, l}\right)=\operatorname{InvGamma}\left(a_{i, l}, b_{i, l}\right)
\end{aligned}
$$

where $\phi=\left(a_{i, l}, b_{i, l}\right)$ are the hyperparameters to optimize over, and $\boldsymbol{\mu}_{\nu}$ and $\boldsymbol{\sigma}_{\nu}$ are calculated as in Eqs. (11)-(12).

\section{EXPERIMENTAL RESULTS}

An experimental test is performed to examine the proposed methodology. Measurements were performed inside a room of approximate dimensions $6 \times 9 \times 3 \mathrm{~m}$ (see Fig. 1). The room has a suspended absorptive ceiling, and acoustically absorptive panels distributed on two of the walls (see white panels in Fig. 1). All walls have scattering elements, panel diffusers, blackboards, whiteboards, etc. One of the walls is highly irregular, having a $40 \mathrm{~cm}$ shelf/protrusion below the window panes used for heating and ventilation and three vertical columns. The room, normally used as a classroom for teaching, is furnished with tables, chairs, blackboards, etc. (see Fig. 1). The average reverberation time of the room is $0.5 \mathrm{~s}$ for the frequency bands of $250 \mathrm{~Hz}$ to $4 \mathrm{kHz}$.

A total of 504 room impulse responses were measured in the room. From these, 48 points are uniformly distributed on the surface of a sphere of radius $0.25 \mathrm{~m}$. Additionally, there are 456 positions that are measured along a line (reference grid) of length $5.4 \mathrm{~m}$, with an inter-spacing of $1 \mathrm{~cm}$ between sensors. The positions along this line serve as the reference true data for evaluating the reconstruction. A robotic arm (UR5, from Universal Robots) was programmed to sequentially move a $1 / 2$ inch measurement microphone (type 4192, from Brüel $\&$ Kjær) to the measured positions. The microphone was attached to the robotic arm via a $50 \mathrm{~cm}$ steel rod, and the 
measurement positions were centered away from the body of the robot to reduce scattering from the robot's pole. The source used was a two-way loudspeaker (Dynaudio BM6), placed approximately at position $(3,3,1.2) \mathrm{m}$ - see Fig. 1 . The room was excited between $20 \mathrm{~Hz}$ and $20 \mathrm{kHz}$ using a 10 seconds long logarithmic sine sweep. The sweep signal was emitted and recorded using a data acquisition card (USB4431, from National Instruments) with a sampling rate of 48000 samples/second. The background noise was measured every $20^{\text {th }}$ measurement to estimate the signal-to-noise ratio.

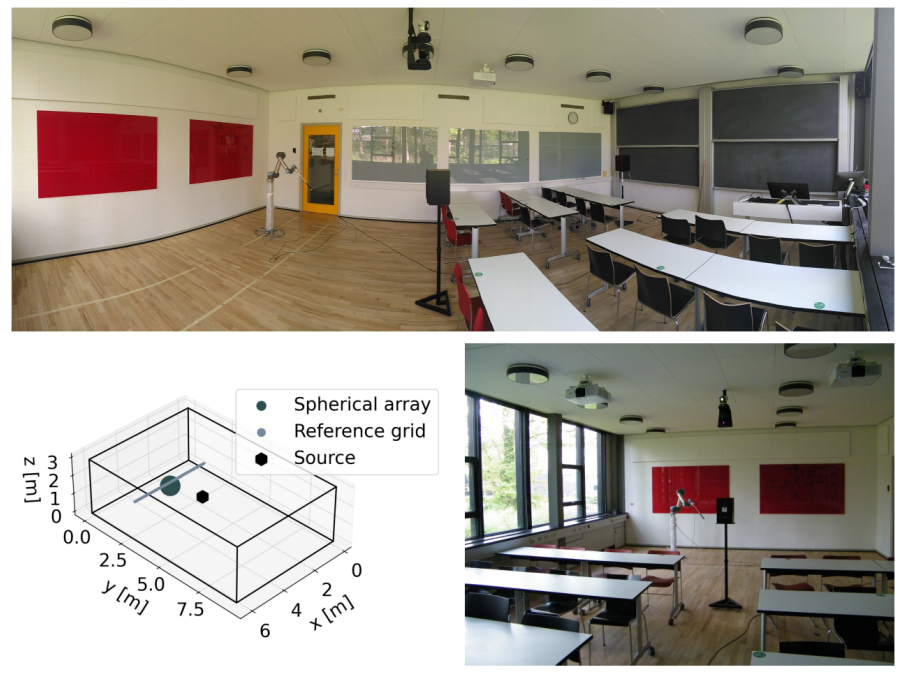

Fig. 1. Experimental setup. Top: Panoramic view of the classroom ( $V=$ $162 \mathrm{~m}^{3}, T_{60} \tilde{5} 00 \mathrm{~ms}$ ) where experiments were performed. Source and robotic arm are visible. Bottom left: Diagram showing the position of the soruce in the room, the position of the spherical array and the reference 'true' pressure line. Bottom right: Front-view of the classroom, showing also the windowed side wall.

The proposed model consists of three main components, corresponding to the three terms of Eq. (6): $D=20$ sources in a Equivalent Source expansion [40], [41] - which could also be a multipole or spherical harmonic expansion as in Ref. [42] to model the direct sound field, positioned on a sphere of radius $10 \mathrm{~cm}$ around the estimated location of the loudspeaker. The choice of $D$ would be one sufficient to model the radiation of the source (directivity and acoustic center), for which $\mathrm{D}=20$ is sufficient in the present study. There are $S=1000$ point sources located on a sphere of radius $r_{\text {early }}=4 \mathrm{~m}$ around the center of the spherical array and outside the reconstruction domain of dimension length $L=5.4 \mathrm{~m}$. Finally $J=1000$ plane waves are scaled on planes at a distance $r_{\text {late }}=7 \mathrm{~m}$ from the center of the spherical array (these planes lie outside the room boundaries). The specific choice of $S$ and $J$ depends mostly on the size of the reconstruction domain, and subject to a compromise between accuracy computational cost, typically considering less than $N=(\lceil\mathrm{e} \pi R / \lambda\rceil+1)^{2}$ [44]. The proposed model was implemented in the probabilistic inference platform Pyro, which allows for efficient inference of the unknown parameters $\sigma_{l, i}^{2}$ via stochastic variational inference as in Section II-D. The

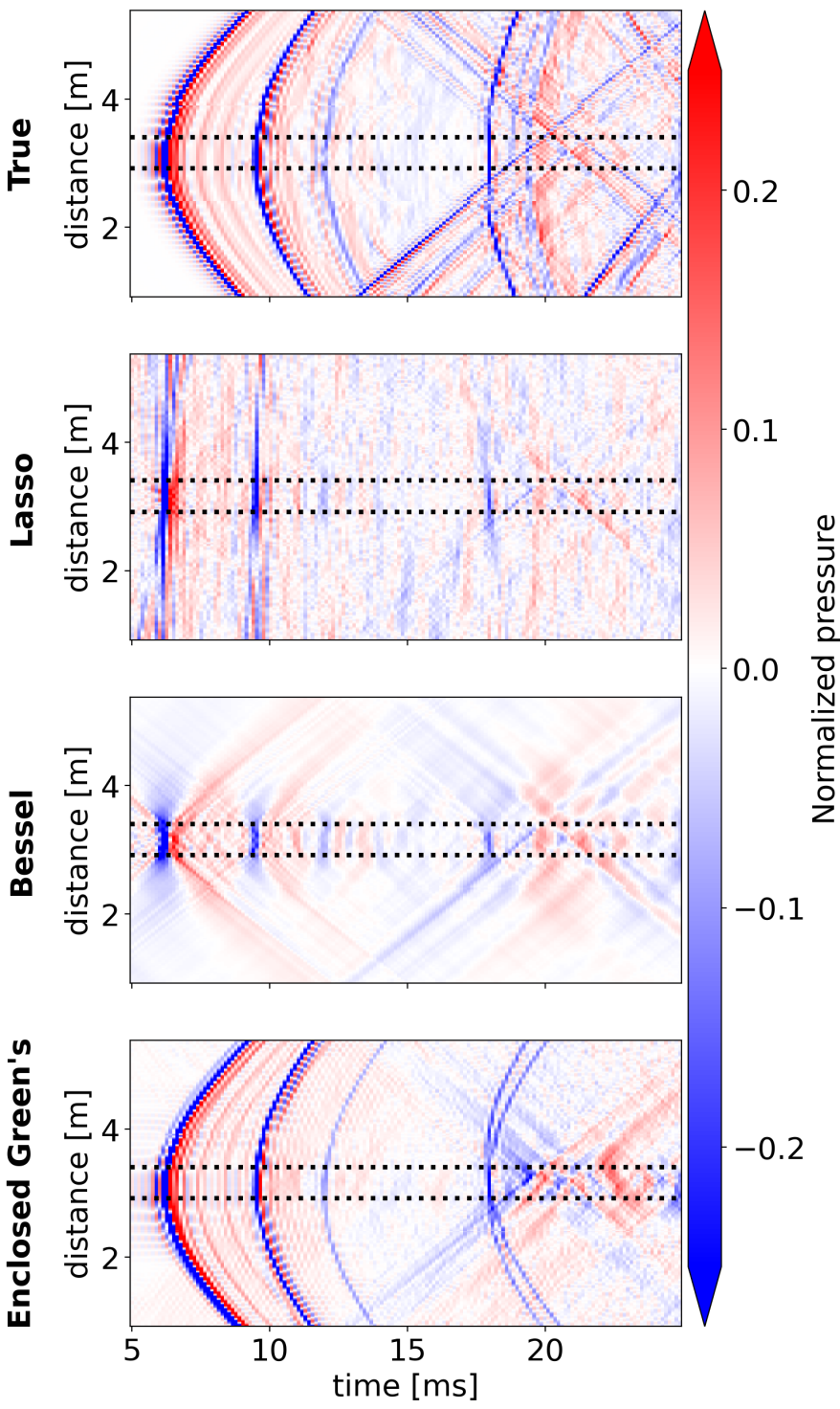

Fig. 2. Space-time sound pressure along a reconstruction aperture of $4.56 \mathrm{~m}$ in the room. The measurement aperture $(50 \mathrm{~cm})$ is indicated by dashed black lines. Top: True pressure field. Center-top: reconstruction with plane wave expansion and Lasso. Center-bottom: Reconstruction with Gaussian process regression and a diffuse field kernel as in Eq. (18). Bottom: Proposed reconstruction with enclosed Green's function and variational Gaussian process [Eq. (6)]

prior distribution over the variances is an inverse gamma

$$
\sigma_{i, l} \sim \operatorname{InvGamma}(30,0.5),
$$

and the measurement noise is estimated from the measurements as independent noise, such that $\kappa_{e}\left(t, \mathbf{r}, t^{\prime}, \mathbf{r}^{\prime}\right)=\sigma_{e}^{2}$.

Figure 2 shows the true measured field and the reconstructed sound fields using 1) Lasso regression with a plane wave expansion in the wavenumber domain with $J=1000]$, 2) a diffuse field (Bessel) kernel Gaussian process as in Eq. (18) [which corresponds theoretically to $J \rightarrow \infty$, and 3) the proposed methodology based on the structure of the Green's Function in an enclosure (denoted in figures 2-5 as EGF 
- for Enclosed Green's Function) as in Eq. (6) [with $D=$ $20, S=1000, J=1000]$. The reconstruction is estimated up to a sampling frequency of $8 \mathrm{kHz}$. The figure shows the reconstruction for the first $25 \mathrm{~ms}$ of the room impulse response. The measurement aperture is $50 \mathrm{~cm}$ (indicated in the figure by the dotted black lines) and the reconstruction is over an aperture of $4.56 \mathrm{~m}$. It should be noted that there are some visible artifacts around the direct sound and early reflections, which are due to downsampling and the graphical interpolation used in the plots. It is apparent that all methods interpolate fairly well within the $50 \mathrm{~cm}$ aperture- i.e. the reconstruction of the sound field within the measurements is correct. However, significant differences between the methods are found when extrapolating the data outside the measurement aperture. The Plane wave expansion with Lasso regularization (Fig. 2b) tends to reconstruct planar wave fronts, so that the temporal structure is somewhat maintained, but the spatial structure of the field is lost in the reconstruction. This is not surprising, as the physical information incorporated into the problem (few sparse planar wave fronts) is not representative of the underlying physical problem. The Bessel/diffuse field kernel (Fig. 2c) fails to reconstruct the direct field and early part of the response, and most early reflections are not reconstructed (e.g. floor, ceiling and back wall). However, as the density of reflections increases (after $20 \mathrm{~ms}$ approximately), the predictions improve, and the overlapping reflections start to be correctly extrapolated. Finally, the proposed enclosed Green's Function (EGF) approach (Fig. 2d) recovers the direct field perfectly (which is modeled as an equivalent source method after localizing the position of the source [45]), and succeeds in extrapolating it over the $4.56 \mathrm{~m}$ aperture. The method is able to recover the scattering by the furniture in the room - scattered components are visible in the trailing wavefronts after the direct field (2-3 ms behind it), before the arrival of the floor reflection at $t=10 \mathrm{~ms}$. The ceiling reflection is also well recovered at $t \approx 13 \mathrm{~ms}$, having less energy due to the dissipation of sound at the absorptive boundary. It is then noticeable, that the reflection from the back-wall is also reconstructed, although in this case it is divided into two closely superimposed spherical wave fronts, since the position of the monopole reconstruction basis (second term in the right hand side of Eq. (6)) starts to differ from the actual spatial divergence of the waves, i.e. due to model mismatch/discrepancy. After this time $(t=20 \mathrm{~ms})$ the reconstruction is performed using the plane wave basis (last term of Eq. (6), which is successful at recovering this part of the response. It should be noted that the improved reconstruction comes at a much increased computational complexity.

Figure 3 shows the averaged temporal correlation between the three reconstruction methods and the true reference field, both for the interpolated data as well as for the extrapolated data in windows of $5 \mathrm{~ms}$. It is apparent from Fig. 3 that the early part of the response is best reconstructed by the proposed enclosed Green's function approach. After $20 \mathrm{~ms}$, the reconstruction using a Gaussian process regression with a Bessel kernel starts to model the pressure field more accurately.
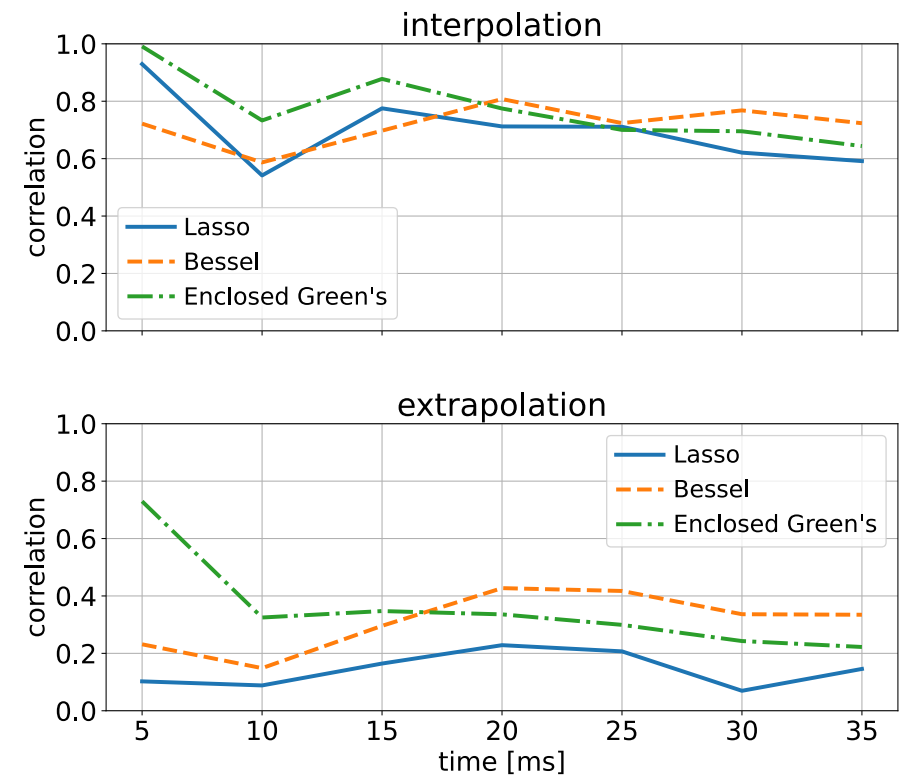

Fig. 3. Correlation coefficient between the reconstructed room impulse responses and the true field over the $4.56 \mathrm{~m}$ reconstruction line. the correlation coefficient is estimated sequentially in $5 \mathrm{~ms}$ time windows, for the Lasso plane wave expansion, the Bessel kernel GP and the proposed Enclosed Green's function GP.

These results also corroborate that such Bessel kernel is wellsuited for reconstructing more isotropic fields, such as the one found in the room after reaching sufficient reflection density. The lowest correlation is obtained with the Lasso plane-wave reconstruction.
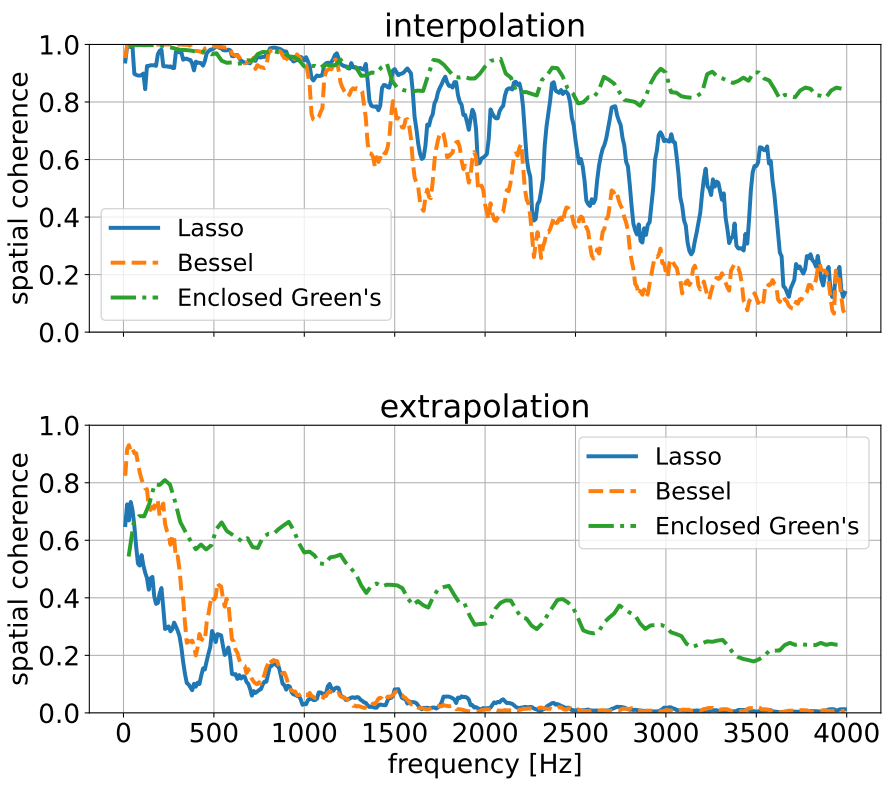

Fig. 4. Spatial coherence between the reconstructed room impulse responses and the true field, over the $4.56 \mathrm{~m}$ reconstruction line, as a funciton of frequency.The methods are Lasso plane wave expansion, the Bessel GP and the proposed Enclosed Green's function GP.

Figure 4 shows the spatial coherence/similarity between the 
reconstructed fields and the true field in frequency domain also for interpolated and extrapolated reconstruction points. The proposed EGF approach is superior in all the frequency range, which is mostly a result of the near-perfect reconstruction of the direct field and early reflections. These contain much of the energy in the response, and the reconstruction of the fine structure in the early response contributes to a proper broadband reconstruction, devoid of coloration effects and the typical roll-offs due to sampling limits.

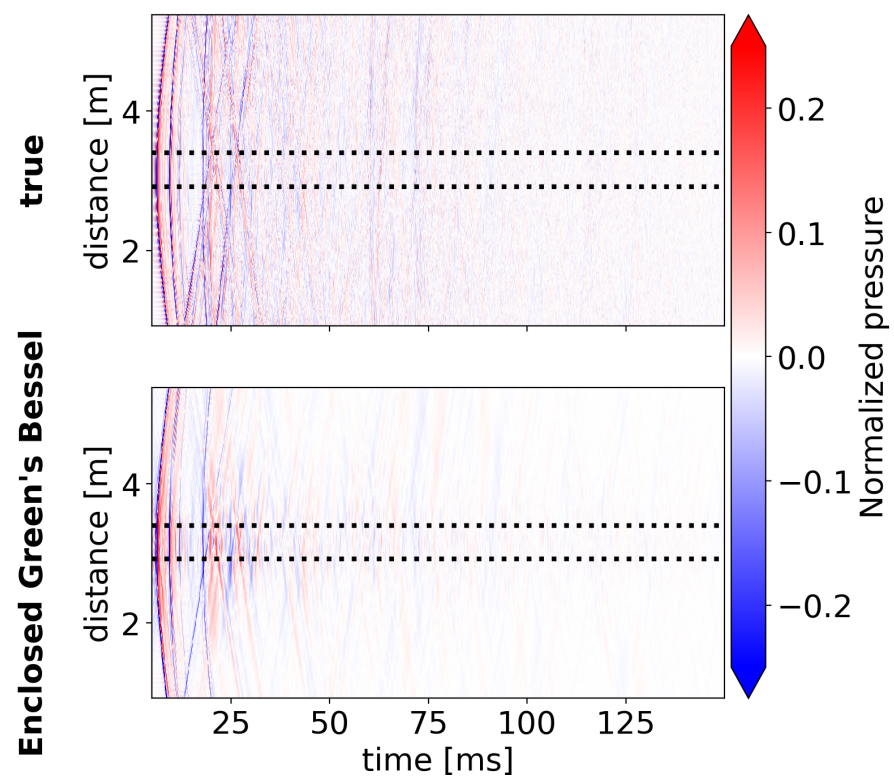

Fig. 5. Time-space reflection chart over reconstruction line, $150 \mathrm{~ms}$. The reconstruciton is performed with the proposed Enclosed Green's function GP [Eq. (6)] for the early part of the response ( $25 \mathrm{~ms})$, and the rest of the response estimated with a diffuse field kernel GP [Eq. (18)].

Based on these findings, it seems sensible to reconstruct the early part of the response with an accurate explicit model (such as the proposed EGF), and the later part with the diffuse field kernel. Such reconstruction is shown in Fig. 5: in the early part (first $25 \mathrm{~ms}$ ) the reconstruction is based on the enclosed Green's Function model - which consists of a highly anisotropic kernel that is well-suited to the spatial properties of the field, and the rest of the response is reconstructed using the Bessel/diffuse kernel [Eq.(18)], which is isotropic and well suited to seemingly spatially random wave fields. The reconstruction shows superior performance to established approaches. It is however visible how the extrapolated data in the late part of the response is somewhat underestimated (lower energy than the true field), which is a result of the properties of the Bessel kernel, which is spatially decaying, and tends to provide low energy estimates when extrapolating further away than half a wavelength distance from the measurements (it should be noted that this is however a very challenging problem: mapping measurements from a $50 \mathrm{~cm}$ aperture into a reconstruction aperture of almost $5 \mathrm{~m}$ ).

\section{CONCLUSIONS}

In this study, a reconstruction method based on modeling the spatio-temporal properties of enclosed fields has been introduced. The approach makes use of the structure of the time domain Green's function in an enclosure. The problem is formulated as a Variational Gaussian process regression. The approach is successful in reconstructing the spatio-temporal response over a large spatial aperture. For the later part of the response, isotropic diffuse kernels are convenient, as their prediction accuracy is similar or greater, but both the model and computational complexity is much lower. Experimental results have been presented to demonstrate that the proposed approach can reconstruct the sound field in a real room and over a large spatial aperture $(4.56 \mathrm{~m}$ aperture $)$ based on localized measurements with a 48-channel spherical array, of radius $25 \mathrm{~cm}$.

\section{ACKNOWLEDGEMENTS}

This work is supported by the VILLUM Foundation, under grant nr. 19179. the authors would like to thank Marco Berzborn for help with the robot scanning and Martin Bo Møller for valuable discussion.

\section{REFERENCES}

[1] I. B. Witew, M. Vorländer, and N. Xiang, "Sampling the sound field in auditoria using large natural-scale array measurements," J. Acoust. Soc. Am., vol. 141, no. 3, pp. EL300-EL306, 2017.

[2] S. A. Verburg and E. Fernandez-Grande, "Reconstruction of the sound field in a room using compressive sensing," J. Acoust. Soc. Am., vol. 143, no. 6, pp. 3770-3779, 2018.

[3] G. Götz, S. J. Schlecht, A. M. Ornelas, and V. Pulkki, "Autonomous robot twin system for room acoustic measurements," J. Audio Eng. Soc., vol. 69, no. 4, pp. 261-272, april 2021.

[4] F. M. Heuchel, D. Caviedes-Nozal, J. Brunskog, F. T. Agerkvist, and E. Fernandez-Grande, "Large-scale outdoor sound field control," The Journal of the Acoustical Society of America, vol. 148, no. 4, pp. 2392 $2402,2020$.

[5] M. Cobos, J. J. Perez-Solano, S. Felici-Castell, J. Segura, and J. M. Navarro, "Cumulative-sum-based localization of sound events in lowcost wireless acoustic sensor networks," IEEE/ACM Transactions on Audio, Speech, and Language Processing, vol. 22, no. 12, pp. 1792 1802, 2014.

[6] Y. Oikawa, M. Goto, Y. Ikeda, T. Takizawa, and Y. Yamasaki, "Sound field measurements based on reconstruction from laser projections," in Proceedings. (ICASSP '05). IEEE International Conference on Acoustics, Speech, and Signal Processing, 2005., vol. 4, 2005, pp. iv/661iv/664 Vol. 4.

[7] A. Torras-Rosell, S. Barrera-Figueroa, and F. Jacobsen, "Sound field reconstruction using acousto-optic tomography," The Journal of the Acoustical Society of America, vol. 131, no. 5, pp. 3786-3793, 2012.

[8] S. Verburg Riezu and E. Fernandez Grande, "Acousto-optic sensing - spatial reconstruction of the sound field enclosed in a room," in Proceedings of the 23rd International Congress on Acoustics, 2019, pp. 4940-4947.

[9] E. Fernandez Grande, S. Verburg Riezu, and M. Hahmann, "Acoustooptic capture of the sound field in a room based on sparse measurement data," in Proceedings of the International Symposium on Room Acoustics. Nederlands Akoestisch Genootschap, 2019, pp. 451-457.

[10] E. G. Williams, Fourier Acoustics - Sound Radiation and Nearfield Acoustical Holography. New York: Academic Press, 1999, Chaps. 2,6 .

[11] E. G. Williams and J. D. Maynard, "Holographic Imaging without the Wavelength Resolution Limit,", vol. 45, no. 7, pp. 554-557, Aug. 1980.

[12] J. D. Maynard, E. G. Williams, and Y. Lee, "Nearfield acoustic holography: I. theory of generalized holography and the development of nah," The Journal of the Acoustical Society of America, vol. 78, no. 4, pp. 1395-1413, 1985. 
[13] E. G. Williams, N. Valdivia, P. C. Herdic, and J. Klos, "Volumetric acoustic vector intensity imager," The Journal of the Acoustical Society of America, vol. 120, no. 4, pp. 1887-1897, 2006.

[14] M. Nolan, S. A. Verburg, J. Brunskog, and E. Fernandez-Grande, "Experimental characterization of the sound field in a reverberation room," The Journal of the Acoustical Society of America, vol. 145, no. 4 pp. 2237-2246, 2019.

[15] J. Garcia-Bonito, S. J. Elliott, and C. C. Boucher, "Generation of zones of quiet using a virtual microphone arrangement," The Journal of the Acoustical Society of America, vol. 101, no. 6, pp. 3498-3516, 1997.

[16] M. Møller, J. Nielsen, E. Fernandez Grande, and S. Olesen, "On the influence of transfer function noise on sound zone control in a room," IEEE/ACM Transactions on Audio, Speech, and Language Processing, vol. 27, no. 9, pp. 1405-1418, Sep. 2019.

[17] J. Tylka and E. Choueiri, "Comparison of techniques for binaural navigation of higher-order ambisonic soundfields," Jan. 2015, 139th Audio Engineering Society International Convention, AES 2015 ; Conference date: 29-10-2015 Through 01-11-2015.

[18] J. G. Tylka and E. Y. Choueiri, "Performance of linear extrapolation methods for virtual sound field navigation," J. Audio Eng. Soc, vol. 68, no. 3, pp. 138-156, 2020. [Online]. Available: http://www.aes.org/elib/browse.cfm?elib $=20725$

[19] — , "Fundamentals of a parametric method for virtual navigation within an array of ambisonics microphones," J. Audio Eng. Soc, vol. 68, no. 3, pp. 120-137, 2020 .

[20] L. Birnie, T. Abhayapala, V. Tourbabin, and P. Samarasinghe, "Mixed source sound field translation for virtual binaural application with perceptual validation," IEEE/ACM Transactions on Audio, Speech, and Language Processing, vol. 29, pp. 1188-1203, 2021.

[21] Müller, Kaspar and Zotter, Franz, "Auralization based on multi-perspective ambisonic room impulse responses," Acta Acust., vol. 4, no. 6, p. 25, 2020. [Online]. Available: https://doi.org/10.1051/aacus/2020024

[22] V. Garcia-Gomez and J. J. Lopez, "Binaural room impulse responses interpolation for multimedia real-time applications," in Audio Engineering Society Convention 144, May 2018. [Online]. Available: http://www.aes.org/e-lib/browse.cfm?elib=19479

[23] R. Mignot, L. Daudet, and F. Ollivier, "Room reverberation reconstruction: interpolation of the early part using compressed sensing," IEEE Trans. Audio, Speech, Lang. Process., vol. 21, no. 11, pp. 2301-2312, 2013.

[24] R. Mignot, G. Chardon, and L. Daudet, "Low frequency interpolation of room impulse responses using compressed sensing," IEEE/ACM Trans. Audio, Speech, Lang. Process., vol. 22, no. 1, pp. 205-216, 2014.

[25] T. Pham Vu, E. Rivet, and H. Lissek, "Low frequency sound field reconstruction in non-rectangular room: A numerical validation," Euronoise 2018 Proceedings, p. 8, 2018.

[26] E. Fernandez Grande, "Sound field reconstruction in a room from spatially distributed measurements," in Proceedings of 23rd International Congress on Acoustics. German Acoustical Society (DEGA), 2019, pp. 4961-68.

[27] N. Antonello, E. De Sena, M. Moonen, P. A. Naylor, and T. van Waterschoot, "Room impulse response interpolation using a sparse spatiotemporal representation of the sound field," IEEE/ACM Transactions on Audio, Speech, and Language Processing, vol. 25, no. 10, pp. 19291941, 2017.

[28] S. Koyama and L. Daudet, "Sparse representation of a spatial sound field in a reverberant environment," IEEE Journal of Selected Topics in Signal Processing, vol. 13, no. 1, pp. 172-184, 2019.

[29] M. Hahmann, S. A. Verburg, and E. Fernandez-Grande, "Analysis of the sound field in a room using dictionary learning," in Proceedings of 23rd International Congress on Acoustics. German Acoustical Society (DEGA), 2019.

[30] H. Kuttruff, Room acoustics. Crc Press, 2016.

[31] E. Fernandez-Grande, "Sound field reconstruction using a spherical microphone array," J. Acoust. Soc. Am., vol. 139, no. 3, pp. 1168-1178, 2016.

[32] F. Jacobsen and P. M. Juhl, Fundamentals of general linear acoustics. John Wiley \& Sons, 2013.

[33] D. Caviedes-Nozal, N. A. Riis, F. M. Heuchel, J. Brunskog, P. Gerstoft, and E. Fernandez-Grande, "Gaussian processes for sound field reconstruction,” J. Acoust. Soc. Am., vol. 149, no. 2, pp. 1107-1119, 2021.
[34] M. Nolan, M. Berzborn, and E. Fernandez-Grande, "Isotropy in decaying reverberant sound fields," Acoustical Society of America. Journal, vol. 148, no. 2, p. 1077-1088, Aug. 2020.

[35] D. Caviedes-Nozal and E. Fernandez-Grande, "Spatio-temporal gaussian process regression for room impulse response interpolation with acoustically informed priors," J. Acoust. Soc. Am., vol. 149, no. 4, pp. A85-A85, 2021.

[36] P. Gerstoft, C. F. Mecklenbräuker, A. Xenaki, and S. Nannuru, "Multisnapshot sparse bayesian learning for doa," IEEE Signal Processing Letters, vol. 23, no. 10, pp. 1469-1473, 2016.

[37] P. M. Morse and H. Feshbach, Theoretical acoustics. McGraw Hill, 1953.

[38] D. Mintzer, "Transient sounds in rooms," J. Acoust. Soc. Am., vol. 22, no. 3, pp. 341-352, 1950

[39] J. B. Allen and D. A. Berkley, "Image method for efficiently simulating small-room acoustics," J. Acoust. Soc. Am., vol. 65, no. 4, pp. 943-950, 1979.

[40] G. H. Koopmann, L. Song, and J. B. Fahnline, "A method for computing acoustic fields based on the principle of wave superposition," The Journal of the Acoustical Society of America, vol. 86, no. 6, pp. 24332438, 1989.

[41] E. Fernandez-Grande, A. Xenaki, and P. Gerstoft, "A sparse equivalent source method for near-field acoustic holography," J. Acoust. Soc. Am. vol. 141, no. 1, pp. 532-542, 2017.

[42] D. Caviedes Nozal, F. Heuchel, J. Brunskog, N. Riis, and E. Fernandez Grande, "A bayesian spherical harmonics source radiation model for sound field control," Acoustical Society of America. Journal, vol. 146, no. 5 , pp. 3425-3435, 2019.

[43] C. M. Bishop, "Approximate Inference," in Pattern Recognition and Machine Learning, 2006, ch. 10.

[44] R. A. Kennedy, P. Sadeghi, T. D. Abhayapala, and H. M. Jones, "Intrinsic limits of dimensionality and richness in random multipath fields," IEEE Transactions on Signal Processing, vol. 55, no. 6, pp. 2542-2556, 2007.

[45] E. Fernandez-Grande and L. Daudet, "Compressive acoustic holography with block-sparse regularization," J. Acoust. Soc. Am, vol. 143, no. 7, 2018. 